\title{
Entirely Harmonic Domain Calculation of Multiphase Nonsinusoidal Steady State
}

\author{
Taku Noda, Member, IEEE, Adam Semlyen, Life Fellow, IEEE, and Reza Iravani, Fellow, IEEE
}

\begin{abstract}
This paper proposes an algorithm for obtaining the periodic steady-state solution of a multiphase network including nonlinear, switching, and frequency dependent elements. Unlike existing methods which deal with nonlinear and switching elements in the time domain, the approach presented is entirely in the harmonic domain. The method will be used for the harmonic analysis of power systems and for steady-state initialization in electromagnetic transient analysis. The algorithm takes rigorously into account the inter-harmonic couplings in the Jacobian matrix of the proposed Newton-Raphson iteration process so that a quadratic convergence rate is achieved. Linear, nonlinear, switching, and frequency dependent elements are modeled in a modular approach, and any network topology can be handled by extending the Modified Nodal Equations approach to the harmonic domain case. First the algorithm is described and then applied to a test case to demonstrate its computational performance.
\end{abstract}

Index Terms-Multiport circuits, nonlinear circuits, periodic functions, power system harmonics, switching circuits.

\section{INTRODUCTION}

$\mathbf{P}$ OWER systems include nonlinear, switching, and frequency dependent elements. Iron cores of transformers, loads with rectifiers, and discharge lamps are nonlinear elements that generate a nonsinusoidal current even if a purely sinusoidal voltage is applied. HVdc converters and power-electronics devices are switching elements that control the currents by semiconductor switch operations. Overhead lines, underground cables, and transformer impedances are frequency dependent elements due to skin effect. To predict the harmonic content and propagation of a network with such elements, an algorithm for calculating the periodic steady-state solution is required. The algorithm can also be used for steady-state initialization in electromagnetic transient analysis.

This field of research was pioneered by Xia and Heydt [1], [2] using a decoupled positive-sequence circuit. Later, Densem et al. pointed out that the network must be modeled in phase coordinates since noncharacteristic harmonics cannot be properly evaluated by a positive-sequence circuit [3]. Subsequently proposed multiphase methods can be classified, in terms of their formulation methodologies, into three categories: harmonic domain methods [4]-[8], time domain methods [9], [10], and hy-

Manuscript received May 10, 2003.

T. Noda is with Electrical Insulation Department, Central Research Institute of Electric Power Industry (CRIEPI), Tokyo 201-8511, Japan (e-mail: takunoda@ieee.org).

A. Semlyen and R. Iravani are with the Department of Electrical and Computer Engineering, University of Toronto, Toronto, ON M5S 3G4, Canada (e-mail: adam.semlyen@utoronto.ca; iravani@ecf.utoronto.ca).

Digital Object Identifier 10.1109/TPWRD.2004.829944 brid methods [11], [12]. The harmonic domain methods deal with all network elements in the harmonic domain. Although the treatment of linear elements including frequency dependent elements is straightforward, that of nonlinear and switching elements which produce couplings between different harmonics is of concern. A practical solution used by most of the existing harmonic domain methods [5]-[8] is to use the values at the previous iteration step to eliminate the harmonic couplings in the formulation at the expense of slower convergence rate (this is basically equivalent to injecting the currents of nonlinear and switching elements obtained at the previous iteration step). On the other hand, the harmonic domain method presented in [4] takes rigorously into account the harmonic couplings based on the state-space representation of the network, but deriving the state equations can be difficult in particular network configurations. Another solution is to use the hybrid methods which deal with nonlinear and switching elements in the time domain while the remaining linear part is treated in the harmonic domain. The time domain methods seem to be at a development stage since the methods are applied only to simple networks.

This paper presents an algorithm to calculate the periodic steady-state solution of a multiphase network including nonlinear, switching, and frequency dependent elements. The approach presented formulates the network equations entirely in the harmonic domain by extending the Modified Nodal Equations approach [13] to the harmonic domain case so that the network equations can always be derived regardless of network topology. The network equations are then solved by a Newton-Raphson iteration process. In a modular approach, the harmonic domain branch constitutive relations and their linearized admittance matrices are derived for lumped linear elements, frequency dependent elements, switching elements, and nonlinear elements. The linearized admittance matrices, which correspond to the individual Jacobian matrices of the network elements, are derived based on the same principle as the method presented in [4]. Thus, the proposed algorithm takes rigorously into account the harmonic couplings and this achieves a quadratic convergence rate. Since the proposed formulation includes network equations for all harmonics, the Jacobian matrix of the entire network can be very large. It is shown that the large Jacobian matrix can be efficiently handled by taking advantage of its sparsity.

The paper first describes the proposed algorithm, and then the steady-state solution of a $500 \mathrm{kV}$ double-circuit three-phase test network including nonlinear inductors, an SVC, and frequency dependent transmission lines is calculated. It is shown that the convergence rate of the proposed algorithm is quadratic. 


\section{HARMONIC DOMAIN NOTATION}

\section{A. Operator of Time Derivative}

The complex Fourier series of a time domain $T$-periodic scalar quantity $x(t)$ is given by

$$
x(t)=\sum_{h=-\infty}^{\infty} x_{h} \mathrm{e}^{j h \omega_{0} t}
$$

where $x_{h}(h=0, \pm 1, \pm 2, \cdots)$ are the Fourier coefficients and $\omega_{0}=2 \pi / T$ is the base angular frequency. Let $y(t)$ be the time derivative of $x(t)$ :

$$
y(t)=\frac{d x(t)}{d t}=\sum_{h=-\infty}^{\infty} j h \omega_{0} x_{h} \mathrm{e}^{j h \omega_{0} t}
$$

where $y(t)$ is also $T$-periodic. Considering harmonics only up to the $\pm K$-th, the coefficients of the exponential terms can be expressed in the following matrix notation:

$$
y=S x
$$

where $x, y \in \mathbf{C}^{M}(M=2 K+1)$ are the harmonic domain vectors corresponding to $x(t)$ and $y(t)$, defined by

$$
x=\left[\begin{array}{c}
x_{-K} \\
\vdots \\
x_{-1} \\
x_{0} \\
x_{1} \\
\vdots \\
x_{K}
\end{array}\right], \quad y=\left[\begin{array}{c}
y_{-K} \\
\vdots \\
y_{-1} \\
y_{0} \\
y_{1} \\
\vdots \\
y_{K}
\end{array}\right]
$$

and $S$ is the diagonal $M \times M$ matrix defined by

$$
S=\operatorname{diag}\left(j k \omega_{0}\right) \quad \text { for } k=-K, \cdots, K .
$$

Thus $S$ is the operator of time derivative in the harmonic domain [4], [14]. Note that $S$ is singular because $k$ includes zero.

\section{B. Incremental Expression for Time Domain Nonlinearity}

Next, we consider the nonlinear algebraic relation between two $T$-periodic scalar variables $x(t)$ and $y(t)$ in the time domain:

$$
y(t)=f(x(t)) .
$$

For incremental changes of $x(t)$ and $y(t)$, (6) is linearized as

$$
\Delta y(t)=f_{x}(t) \Delta x(t),
$$

where $f_{x}(t)$ is the derivative of $f(\cdot)$ with respect to $x(t)$. Thus $f_{x}(t)$ is also $T$-periodic. Let $\Delta x$ and $\Delta y$ be the harmonic domain counterparts of $\Delta x(t)$ and $\Delta y(t)$ defined similarly to $x$ and $y$ in (4). The harmonic domain relation for (7) is

$$
\Delta y=F \Delta x
$$

where $F$ is an $M \times M$ Hermitian Toeplitz matrix consisting of the Fourier coefficients $c_{k}$ of $f_{x}(t)$ up to the $\pm K$-th harmonic [4], [14]. Thus, for $K=3, F$ takes the form

$$
F=\left[\begin{array}{lllllll}
c_{0} & c_{1}^{*} & c_{2}^{*} & c_{3}^{*} & & & \\
c_{1} & c_{0} & c_{1}^{*} & c_{2}^{*} & c_{3}^{*} & & \\
c_{2} & c_{1} & c_{0} & c_{1}^{*} & c_{2}^{*} & c_{3}^{*} & \\
c_{3} & c_{2} & c_{1} & c_{0} & c_{1}^{*} & c_{2}^{*} & c_{3}^{*} \\
& c_{3} & c_{2} & c_{1} & c_{0} & c_{1}^{*} & c_{2}^{*} \\
& & c_{3} & c_{2} & c_{1} & c_{0} & c_{1}^{*} \\
& & & c_{3} & c_{2} & c_{1} & c_{0}
\end{array}\right] .
$$

Here the relation $c_{-k}=c_{k}^{*}$ (complex conjugate) is used.

\section{Half-Wave Symmetry}

If the driving force of a network is purely sinusoidal and if all nonlinear elements and periodically-operating switches result in only even order coefficients in matrix (9), all voltage and current waveforms in the network exhibit "half-wave symmetry"; there are no even harmonics in the voltage and current waveforms and no odd harmonics in the coefficient matrices. This is the usual case for ac power networks. If the network of interest exhibits this half-wave symmetry, the time domain derivative (3) and multiplication (8), expressed in the harmonic domain, can be compacted so that $x, y, S$, and $F$ are given by (for $K=3$ )

$$
\begin{aligned}
x & =\left[\begin{array}{c}
x_{-3} \\
x_{-1} \\
x_{1} \\
x_{3}
\end{array}\right], \quad y=\left[\begin{array}{c}
y_{-3} \\
y_{-1} \\
y_{1} \\
y_{3}
\end{array}\right] \\
S & =\operatorname{diag}\left(-j 3 \omega_{0},-j \omega_{0}, j \omega_{0}, j 3 \omega_{0}\right) \\
F & =\left[\begin{array}{llll}
c_{0} & c_{2}^{*} & & \\
c_{2} & c_{0} & c_{2}^{*} & \\
& c_{2} & c_{0} & c_{2}^{*} \\
& & c_{2} & c_{0}
\end{array}\right] .
\end{aligned}
$$

In this case, $S$ is not singular and $M=K+1$.

\section{Operator of Time Integral}

The operator of time integral in the harmonic domain is given by $S^{-1}$ in case of half-wave symmetry. Otherwise, the inverse of $S$ cannot be taken, and therefore we use the Moore-Penrose pseudoinverse $S^{+}$defined by

$$
S^{+}=\operatorname{diag}\left(\cdots,-\frac{1}{j 2 \omega_{0}},-\frac{1}{j \omega_{0}}, 0, \frac{1}{j \omega_{0}}, \frac{1}{j 2 \omega_{0}}, \cdots\right) .
$$

Note that $S^{+}$ignores the dc component. Thus, if $S^{+}$is used, a dc component would not be properly calculated.

\section{E. HDAF and HDAM}

In this paper, the harmonic domain nonlinear function $f(\cdot)$ between the vectors $i$ and $v$ of the branch current and voltage of a network element:

$$
i=f(v)
$$


is called HDAF (Harmonic Domain Admittance Function). The harmonic domain matrix $Y$ that relates to each other the incremental vectors $\Delta i$ and $\Delta v$ of the branch current and voltage:

$$
\Delta i=Y \Delta v
$$

is called HDAM (Harmonic Domain Admittance Matrix). In the case of a single-phase network element, the harmonic domain vectors $i, v, \Delta i$, and $\Delta v$ take the form (4), $f(\cdot) \in \mathbf{C}^{M} \rightarrow \mathbf{C}^{M}$, and $Y \in \mathbf{C}^{M \times M}$. In the case of a multiphase element (the three-phase case is shown throughout the paper for example of the multiphase case), the branch current and voltage vectors take the form

$$
i=\left[\begin{array}{l}
{\left[i_{a}\right]} \\
{\left[i_{b}\right]} \\
{\left[i_{c}\right]}
\end{array}\right], \quad v=\left[\begin{array}{l}
{\left[v_{a}\right]} \\
{\left[v_{b}\right]} \\
{\left[v_{c}\right]}
\end{array}\right]
$$

where the subvectors $\left[i_{a}\right],\left[i_{b}\right],\left[i_{c}\right],\left[v_{a}\right],\left[v_{b}\right]$, and $\left[v_{c}\right]$ are in the form (4). The HDAF is $f(\cdot) \in \mathbf{C}^{3 M} \rightarrow \mathbf{C}^{3 M}$, and the HDAM is expressed as

$$
Y=\left[\begin{array}{lll}
{\left[Y_{a a}\right]} & {\left[Y_{a b}\right]} & {\left[Y_{a c}\right]} \\
{\left[Y_{b a}\right]} & {\left[Y_{b b}\right]} & {\left[Y_{b c}\right]} \\
{\left[Y_{c a}\right]} & {\left[Y_{c b}\right]} & {\left[Y_{c c}\right]}
\end{array}\right] \in \mathbf{C}^{3 M \times 3 M}
$$

where $\left[Y_{i j}\right] \in \mathbf{C}^{M \times M}(i, j=a, b, c)$.

In the proposed method, we use a modular approach [8], [12] where the HDAFs and HDAMs of all network elements are individually calculated and then assembled to form the global HDAF and HDAM of the whole network to be used for a Newton-Raphson iteration.

\section{HDAFs AND HDAMS OF NETWORK ELEMENTS}

The proposed method is built on the types of passive network elements shown in Fig. 1 where the reference directions of the branch voltages and currents are defined. The network elements whose HDAFs and HDAMs are derived below are classified into (a) or (b) of Fig. 1.

Because in the case of a linear network element the incremental current-voltage relation $\Delta i=Y \Delta v$ is also satisfied by the full current-voltage relation $i=Y v$, the HDAF is readily obtained by the HDAM. Thus only HDAMs are derived for linear elements. For nonlinear elements both HDAFs and HDAMs are derived.

\section{A. Single-Phase Linear $R, L, C$ Elements}

Using the operators of time derivative and integral, the HDAMs of the single-phase linear time-invariant resistor $R$, inductor $L$, and capacitor $C$ are respectively given by

$$
Y_{R}=\left(\frac{1}{R}\right) I, \quad Y_{L}=\left(\frac{1}{L}\right) S^{-1}, \quad Y_{C}=C S
$$

where $I$ is the $M \times M$ identity matrix. Because those network elements do not have inter-harmonic couplings, their HDAMs are diagonal matrices. The expressions for the inductor and the capacitor are valid in case of half-wave symmetry.

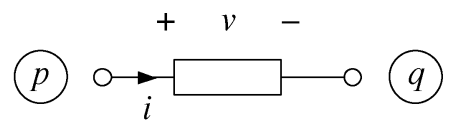

(a)

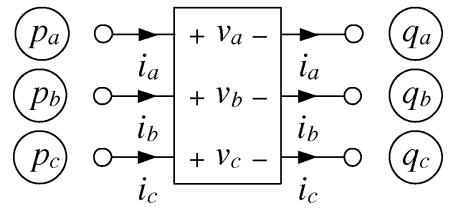

(b)

Fig. 1. The types of passive network elements, considered in the proposed algorithm, with their reference directions of the branch voltages and currents. The above elements are connected from the $p$-th to the $q$-th node where the letters $p$ and $q$ enclosed in circles are node indices. In (b) the three-phase case is shown. (a) Single-phase element; (b) multiphase element.

If the network does not exhibit half-wave symmetry, a small resistance $r_{0}$ which is effective only for the dc component is inserted in series to the inductor:

$$
Y_{L}=\left(\frac{1}{L}\right) S^{+}+G_{0 L}
$$

where the $(K+1, K+1)$ element of $G_{O L}$ is $1 / r_{0}$ and the remaining elements are all zeros. Similarly, a small conductance $g_{0}$, effective only for $\mathrm{dc}$, is connected in parallel to the capacitor:

$$
Y_{C}=C S+G_{0 C}
$$

where the $(K+1, K+1)$ element of $G_{0 C}$ is $g_{0}$ and the remaining elements are zeros. Instead of using the small resistance, if the inductor is accompanied by the series resistor $R$, we consider the two elements as one:

$$
Y_{L R}=(L S+R I)^{-1} \text {. }
$$

If the capacitor is accompanied by the parallel conductance $G$, the two elements are again considered as one:

$$
Y_{C G}=C S+G I
$$

\section{B. Single-Phase Linear Frequency Dependent Elements}

We assume that the characteristic of a single-phase linear frequency dependent element is given by the frequency-scan data $Y_{k}$ of its admittance at discrete frequency points $\omega=k \omega_{0}(k=$ $0,1,2, \cdots)$. The HDAM of the frequency dependent element is given by the following formulas. In case of no half-wave symmetry:

$$
Y_{F D}=\operatorname{diag}\left(Y_{k}\right) \quad \text { for } k=-K, \cdots, K
$$

In case of half-wave symmetry:

$$
Y_{F D}=\operatorname{diag}\left(\cdots, Y_{-3 k}, Y_{-k}, Y_{k}, Y_{3 k}, \cdots\right)
$$

where $Y_{-k}=Y_{k}^{*}$.

\section{Multiphase Linear Frequency Dependent Elements}

We assume that the characteristic of a multiphase linear frequency dependent element is also given by the frequency-scan 
data of its admittance matrix at discrete frequency points $\omega=$ $k \omega_{0}(k=0,1,2, \cdots)$ :

$$
\left[\begin{array}{ccc}
Y_{a a, k} & Y_{a b, k} & Y_{a c, k} \\
Y_{b a, k} & Y_{b b, k} & Y_{b c, k} \\
Y_{c a, k} & Y_{c b, k} & Y_{c c, k}
\end{array}\right]
$$

If the submatrices $\left[Y_{i j}\right](i, j=a, b, c)$ of (17) are obtained as shown below, (17) gives the HDAM of the multiphase frequency dependent element. In case of no half-wave symmetry:

$$
\left[Y_{i j}\right]=\operatorname{diag}\left(Y_{i j, k}\right) \quad \text { for } k=-K, \cdots, K .
$$

In case of half-wave symmetry:

$$
\left[Y_{i j}\right]=\operatorname{diag}\left(\cdots, Y_{i j,-3}, Y_{i j,-1}, Y_{i j, 1}, Y_{i j, 3}, \cdots\right) .
$$

In the above two equations, $Y_{i j, k}$ in the right-hand sides are given by the elements of (25).

\section{Multiphase Linear R, L Elements}

If the following admittance matrix is used for (25), (17) gives the HDAM of a multiphase linear series $R L$ circuit:

$$
\left(\left[\begin{array}{lll}
R_{a a} & R_{a b} & R_{a c} \\
R_{b a} & R_{b b} & R_{b c} \\
R_{c a} & R_{c b} & R_{c c}
\end{array}\right]+j k \omega_{0}\left[\begin{array}{lll}
L_{a a} & L_{a b} & L_{a c} \\
L_{b a} & L_{b b} & L_{b c} \\
L_{c a} & L_{c b} & L_{c c}
\end{array}\right]\right)^{-1} .
$$

\section{E. Periodically-Switching Elements}

Power electronics controllers are periodically-switching elements synchronized with the power frequency. Let us consider a switching device which controls the current of a series inductor as shown in Fig. 2. In the time domain, the current-voltage relation of the element is expressed by

$$
i(t)=p(t) \frac{1}{L} \int\{p(t) v(t)\} d t
$$

where the $T$-periodic function $p(t)$ defines the switching instances by giving the value 1 when the switching device is on and 0 when it is off. We first focus on the integrand

$$
v_{L}(t)=p(t) v(t)
$$

Since this is a time-domain multiplication of two variables same as (7), the procedure from (7) to (8) can be used to obtain the corresponding harmonic domain expression

$$
v_{L}=P v
$$

where $P$ is an $M \times M$ Hermitian Toeplitz matrix consisting of the Fourier coefficients ${ }^{1}$ of $p(t)$ taking the form of (9) (note that the Fourier coefficients can be derived analytically).

Applying the above to each multiplication in (29), we finally obtain the HDAM

$$
Y_{s w}=P\left(\frac{1}{L}\right) S^{-1} P
$$

\footnotetext{
${ }^{1}$ The high order Fourier coefficients of $p(t)$ are generally large because of switch operations, and thus the truncation of the Fourier coefficients causes an error corresponding to the Gibbs phenomenon. The error can be reduced by the application of the Sigma Factor window [15] (See Appendix-A).
}

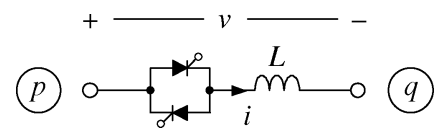

Fig. 2. Switching device which controls the current of the series inductor $L$. This element is connected from the $p$-th to the $q$-th node where the letters $p$ and $q$ enclosed in circles are node indices.

(this is for the half-wave symmetry case and $S^{-1}$ is replaced by $S^{+}$in case of no half-wave symmetry). Finally, we generalize (32) to

$$
Y_{s w}=P Y_{x} P
$$

where $Y_{x}$ is the HDAM of the element whose current is controlled by the switching device. Since (33) is for full values of $i$ and $v$ unlike (7), the HDAF is readily obtained by $i=Y_{s w} v$. To calculate $P$, the switching instants have to be known. However, the on/off instants of a switching element depend on the current and voltage which are not known at this stage. Thus, we introduce the approximation that the on/off instants are determined using the solution at the previous iteration step. The approximation slightly derates the quadratic convergence of a Newton-Raphson iteration, since the on/off instants are determined like a fixed-point iteration procedure. With this approximation, we can sequentially determine the on/off instants using the information of the circuit condition at the previous iteration step and the logic of the control system associated with the switching device.

Instead of introducing the above approximation, it is possible to derive the HDAF and HDAM of a switching device considering the circuit condition. This achieves complete quadratic convergence, but the HDAF and the HDAM have to be derived for each switching device and the HDAF cannot be obtained readily by the HDAM. Reference [16] derives the HDAFs/HDAMs of SVCs and TCSCs.

Coarsely approximated values of the switching instants can be obtained by a formula which gives the behavior of the switching device at the power frequency assuming a balanced condition. At the first iteration step, these values can be used as the initial guess to calculate $P$. At each iteration step, the HADF and HDAM of a switching device are updated as described above.

\section{F. Nonlinear R, L, C Elements}

Because the time domain current-voltage relation of a nonlinear resistor can be written as $i(t)=f(v(t))$ as in (6), the derivation of the HDAM simply follows the procedure from (6) to (9). Thus the HDAM $Y_{N R}$ of the nonlinear resistor is obtained in the form (9), where the Fourier coefficients of a $T$-periodic function, obtained by linearizing $f(\cdot)$ with respect to $v(t)$, are the entries of $Y_{N R}$. To get the HDAF expression of the nonlinear resistor, we symbolically denote the process for obtaining the harmonic domain vectors $i$ and $v$ of a $T$-periodic branch current $i(t)$ and voltage $v(t)$ by $i=\mathcal{F}\{i(t)\}$ and $v=\mathcal{F}\{v(t)\}$ where $\mathcal{F}$ stands for the coefficients of the exponential Fourier series. Using this we obtain the HDAF:

$$
i=\mathcal{F}\left\{f\left(\mathcal{F}^{-1}\{v\}\right)\right\} .
$$


The dynamics of a nonlinear inductor is described by

$$
v(t)=\frac{d \phi(t)}{d t}, \quad i(t)=f(\phi(t))
$$

where $\phi(t)$ is the flux. The procedure from (1) to (3) applies to the first equation to get $v=S \phi$, or for incremental values of $v$ and $\phi$,

$$
\Delta v=S \Delta \phi
$$

The procedure from (6) to (9) applies to the second equation of (35) to get

$$
\Delta i=F \Delta \phi
$$

where $F$ takes the form (9). The entries of $F$ are the Fourier coefficients of a $T$-periodic function obtained by linearizing $f(\cdot)$ with respect to $\phi(t)$. In case of half-wave symmetry, eliminating $\Delta \phi$ from (36) and (37) gives the HDAM

$$
Y_{N L}=F S^{-1}
$$

and the HDAF is

$$
i=\mathcal{F}\left\{f\left(\mathcal{F}^{-1}\left\{S^{-1} v\right\}\right)\right\} .
$$

In case of no half-wave symmetry, $S^{-1}$ is replaced with $S^{+}$ in (38) and (39) and a small resistance $r_{0}$, which is effective only for the dc component, is inserted in series. The HDAM and HDAF are

$$
\begin{aligned}
Y_{N L} & =F S^{+}+G_{0 L}, \\
i & =\mathcal{F}\left\{f\left(\mathcal{F}^{-1}\left\{S^{+} v\right\}\right)\right\}+G_{0 L} v .
\end{aligned}
$$

The dynamics of a nonlinear capacitor is described by

$$
i(t)=\frac{d q(t)}{d t}, \quad q(t)=f(v(t))
$$

where $q(t)$ is the charge. Applying the procedures from (1) to (3) and from (6) to (9) to the above equations, we obtain $\Delta i=$ $S F \Delta v$. Therefore, in case of half-wave symmetry, the HDAM and the HDAF of the nonlinear capacitor are

$$
\begin{aligned}
Y_{N C} & =S F, \\
i & =S \mathcal{F}\left\{f\left(\mathcal{F}^{-1}\{v\}\right)\right\} .
\end{aligned}
$$

In case of no half-wave symmetry, $S^{-1}$ is replaced with $S^{+}$and a small conductance $g_{0}$, effective only for dc, is connected in parallel. The HDAM and HDAF are

$$
\begin{aligned}
Y_{N C} & =S F+G_{0 C}, \\
i & =S \mathcal{F}\left\{f\left(\mathcal{F}^{-1}\{v\}\right)\right\}+G_{0 C} v .
\end{aligned}
$$

\section{SOLUTION PROCESS}

\section{A. Modified Nodal Equations in the Harmonic Domain}

We extend the Modified Nodal Equations approach proposed by Ho et al. [13] to the harmonic domain case and then the harmonic domain network equations are solved to obtain the periodic steady-state solution. Consider a network with $N_{n}$ nodes. The network contains $N_{v}$ independent voltage sources and some independent current sources, whose reference directions for the voltages and currents are defined as in Fig. 3. First, the unknown vector $z$ is defined as

$$
z=\left[\begin{array}{c}
{\left[v_{1}\right]} \\
{\left[v_{2}\right]} \\
\vdots \\
{\left[v_{N_{n}}\right]} \\
{\left[i_{1}\right]} \\
\vdots \\
{\left[i_{N_{v}}\right]}
\end{array}\right] \in \mathbf{C}^{N}
$$

where $N=M\left(N_{n}+N_{v}\right)$. The subvectors $v_{n}(n=$ $\left.1,2, \cdots, N_{n}\right)$ are the harmonic domain vectors of the node voltages, and $i_{n}\left(n=1,2, \cdots, N_{v}\right)$ are those of the currents through the independent voltage sources. Each of the subvectors is a harmonic domain vector which takes the form (4).

Consider a node, in the network, to which passive network elements, independent voltage sources, and independent current sources are connected. The passive elements include all kinds of elements whose HDAFs and HDAMs are given in Section III. The harmonic domain KCL (Kirchhoff's Current Law) for each node is written as

$$
\sum_{n} f_{n}\left(v_{b, n}\right)+\sum_{n} i_{n}=-\sum_{n} i_{s, n} .
$$

The first term of the left-hand side is the sum of the currents leaving the node through the passive elements whose HDAFs $f_{n}(\cdot)$ are described as function of the branch voltages $v_{b, n}$ (in the case of a multiphase element, $f_{n}(\cdot)$ represents a part of the HDAF for the corresponding phase). The second term is the sum of the currents $i_{n}$ leaving the node through the independent voltage sources. The right-hand side term is the sum of the currents $i_{s, n}$ leaving through the independent current sources, where the Fourier coefficients of the waveform $i_{s, n}(t)$ specified for the $n$-th independent current source give the elements of $i_{s, n}$ in the form (4). For the $n$-th independent voltage source connected from the $p$-th to the $q$-th node, we have the following additional branch equations:

$$
v_{p}-v_{q}=v_{s, n}
$$

where $v_{p}$ and $v_{q}$ are the node voltages at $p$ and $q$ respectively, and $v_{s, n}$ consists of the Fourier coefficients of the waveform $v_{s, n}(t)$ specified for the $n$-th independent voltage source.

Since (48) and (49) are harmonic domain expressions, each expression contains $M$ equations. Thus, if we put (48) for all nodes and (49) for all independent voltage sources into a single vector equation, we obtain Modified Nodal Equations in the harmonic domain, namely a global HDAF, containing $N$ equations altogether:

$$
f(z)=-u
$$

where $f(\cdot) \in \mathbf{C}^{N} \rightarrow \mathbf{C}^{N}$ and $u \in \mathbf{C}^{N}$. In the above equation, the first term of the left-hand side in (48) is considered as function of the node voltages because the branch voltages $v_{b, n}$ are the differences of two node voltages. The vector $u$ in (50) is obtained by the right-hand side expressions of (48) and (49). 


\section{B. Newton-Raphson Iteration}

Applying the Newton-Raphson algorithm to (50), we obtain the following iteration process:

$$
z^{(m)}=z^{(m-1)}+\Delta z^{(m)}, \quad \text { for } m=1,2,3, \cdots .
$$

The correction term $\Delta z^{(m)}$ is given by the solution of

$$
J\left(z^{(m-1)}\right) \Delta z^{(m)}=-\left\{f\left(z^{(m-1)}\right)+u\right\}
$$

where

$$
J\left(z^{(m)}\right)=\left.\frac{\partial f}{\partial z}\right|_{z=z^{(m)}}
$$

is the Jacobian matrix, namely the global HDAM. If an appropriate initial guess is not known, $z^{(0)}$ obtained as the solution of $J(0) z^{(0)}=-u$, with $J(0)$ constructed assuming $z=0$, can be used for the initial solution.

\section{Jacobian Matrix}

As explained in [13], the Jacobian matrix $J$ in (53) can be formed by adding and subtracting the "stamps" of all network elements to and from appropriate portions of $J$.

For a passive single-phase element connected from the $p$-th to the $q$-th node [Fig. 1(a)], the HDAM is added to the following two diagonal submatrices of $J$, one submatrix corresponding to the rows of the $p$-th node and the columns of the node voltage $v_{p}$, and the other submatrix corresponding to the rows of the $q$-th node and the columns of the node voltage $v_{q}$. Hereafter, we denote the former submatrix by $J\{p, p\}$ and the latter by $J\{q, q\}$. Also, we subtract the HDAM from the two off-diagonal matrices $J\{p, q\}$ and $J\{q, p\}$. This procedure is illustrated by the stamps shown in Table I(a).

For a passive multiphase element connected from the $p_{a}$-th, $p_{b}$-th, and $p_{c}$-th nodes to the $q_{a}$-th, $q_{b}$-th, and $q_{c}$-th nodes [Fig. 1(b)], the submatrices $Y_{i j}(i, j=a, b, c)$ of the HDAM in (17) are added to and subtracted from the submatrices of $J$ as in Table $\mathrm{I}(\mathrm{b})$.

For an independent voltage source connected from the $p$-th to the $q$-th node [Fig. 3(a)], the $M \times M$ identity matrix $I$ is added to the submatrices $J\{p, n\}$ and $J\{n, p\}$, and subtracted from $J\{q, n\}$ and $J\{n, q\}$ as in Table I(c). The rows denoted by $n$ correspond to the additional branch equations (49) of the voltage source, and the columns denoted by $n$ correspond to the branch current $i_{n}$ through the voltage source [RHS: the right-hand side vector $u$ in (50)].

Starting with the $N \times N$ zero matrix, we scan all passive network elements (single-phase and multiphase) and all independent voltage sources to include their contributions as described in the above, and we finally obtain the complete Jacobian matrix $J$. Also, note the following remarks.

- Because the voltages and currents of independent current sources are not included in the unknown vector $z$, they do not contribute to $J$.

- If one of the nodes $p$ and $q$ is grounded, corresponding rows and columns in Table I are eliminated.

- Controlled sources, which change their voltage or current in proportion to the voltage or current of another branch, can be dealt with as described in [17].
TABLE I

STAMPS FOR PASSIVE NETWORK ELEMENTS AND INDEPENDENT VOLTAGE Sources: (a) Single-Phase ElEMENT; (b) Multiphase ElemENT (ThreE-Phase CASE); (c) IndePendent Voltage Source

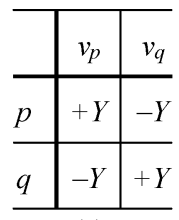

(a)

\begin{tabular}{c|c|c|c|c|c|c}
\hline & $v_{p a}$ & $v_{p b}$ & $v_{p c}$ & $v_{q a}$ & $v_{q b}$ & $v_{q c}$ \\
\hline$p_{a}$ & $+Y_{a a}$ & $+Y_{a b}$ & $+Y_{a c}$ & $-Y_{a a}$ & $-Y_{a b}$ & $-Y_{a c}$ \\
\hline$p_{b}$ & $+Y_{b a}$ & $+Y_{b b}$ & $+Y_{b c}$ & $-Y_{b a}$ & $-Y_{b b}$ & $-Y_{b c}$ \\
\hline$p_{c}$ & $+Y_{c a}$ & $+Y_{c b}$ & $+Y_{c c}$ & $-Y_{c a}$ & $-Y_{c b}$ & $-Y_{c c}$ \\
\hline$q_{a}$ & $-Y_{a a}$ & $-Y_{a b}$ & $-Y_{a c}$ & $+Y_{a a}$ & $+Y_{a b}$ & $+Y_{a c}$ \\
\hline$q_{b}$ & $-Y_{b a}$ & $-Y_{b b}$ & $-Y_{b c}$ & $+Y_{b a}$ & $+Y_{b b}$ & $+Y_{b c}$ \\
\hline$q_{c}$ & $-Y_{c a}$ & $-Y_{c b}$ & $-Y_{c c}$ & $+Y_{c a}$ & $+Y_{c b}$ & $+Y_{c c}$ \\
\hline
\end{tabular}

(b)

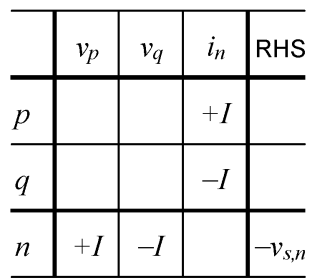

(c)

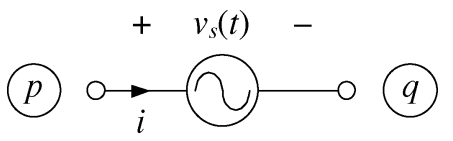

(a)

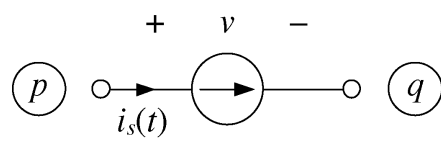

(b)

Fig. 3. The independent voltage and current sources, considered in the proposed algorithm, with their reference directions of the branch voltages and currents. The above elements are connected from the $p$-th to the $q$-th node where the letters $p$ and $q$ enclosed in circles are node indices. In (a) $v_{s}(t)$ is given, and in (b) $i_{s}(t)$ is given. (a) Independent voltage source; (b) independent current source.

\section{Values of $r_{0}$ and $g_{0}$}

When the network does not exhibit half-wave symmetry, we use a small series resistance $r_{0}$ and a small parallel conductance $g_{0}$ for modeling inductors and capacitors respectively. Smaller values of $r_{0}$ and $g_{0}$ give more accurate behavior of the inductors and the capacitors for the dc component. However, too small values will result in an ill-conditioned Jacobian matrix, and thus the values should be determined considering the machine epsilon of the computer being used. ${ }^{2}$

\footnotetext{
${ }^{2}$ If we decide to preserve 10 digits for obtaining the solution using a 16-digit floating-point processor, the largest impedance (in the network) multiplied by $10^{-6}$ is set to $r_{0}$ and the largest admittance multiplied by $10^{-6}$ to $g_{0}$.
} 


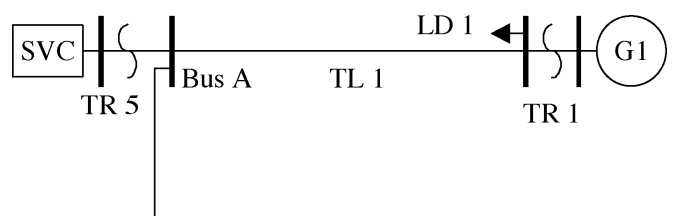

TL 5
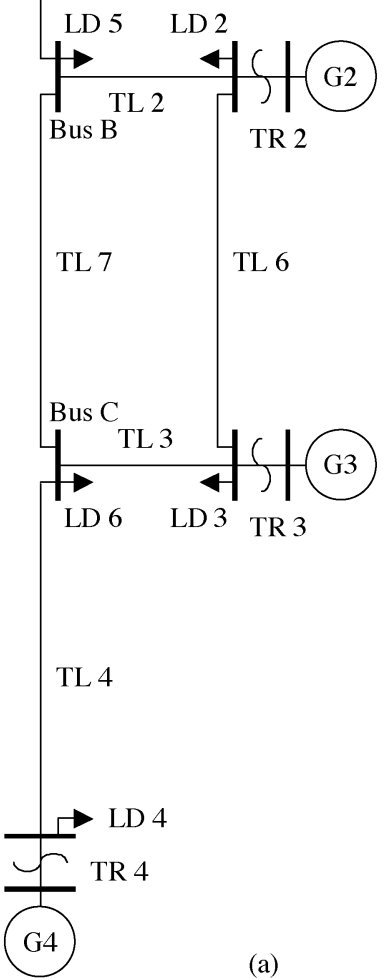

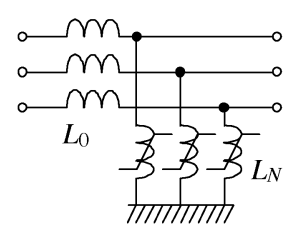

(b)

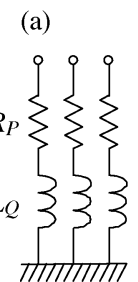

(c)

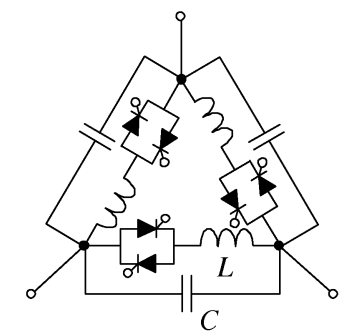

(d)

Fig. 4. $500 \mathrm{kV}$ double-circuit three-phase test network. (a) Network diagram; (b) transformer; (c) load; (d) SVC.

\section{TEST NETWORK}

The periodic steady-state solution of the test network shown in Fig. 4 is calculated by the proposed algorithm. The test network is a $500 \mathrm{kV}$ double-circuit transmission system. The parameter values used in the simulation are given in Table II. Al-
TABLE II

PARAMETER VALUES USED IN THE SimULATION

Generators: the voltages of the generators (500 kV base).

G1: $1.01 \angle 23.91^{\circ}[\mathrm{pu}] \quad$ G2: $1.03 \angle 5.07^{\circ}[\mathrm{pu}]$

G3: $1.03 \angle 3.14^{\circ}[\mathrm{pu}] \quad \mathrm{G} 4: 1.03 \angle 0.00^{\circ}[\mathrm{pu}]$

Transformers: the leakage inductances $L_{0}$ of the transformers. TR1: $10.6[\mathrm{mH}] \quad$ TR2: $15.3[\mathrm{mH}] \quad$ TR3: $15.3[\mathrm{mH}]$ TR4: $5.17[\mathrm{mH}] \quad$ TR5: $6.63[\mathrm{mH}]$

Transmission Lines: the lengths of the transmission lines.

TL1, TL4, TL5, TL6, TL7: 100 [km]

TL2, TL3: $50[\mathrm{~km}]$

Loads: the resistance $R_{P}$ and inductance $L_{Q}$ values of the loads.

LD1: $58.0[\Omega], 57.4[\mathrm{mH}] \quad$ LD2: $86.6[\Omega], 86.6[\mathrm{mH}]$

LD3: $85.1[\Omega], 89.4[\mathrm{mH}] \quad$ LD4: $14.8[\Omega], 6.71[\mathrm{mH}]$

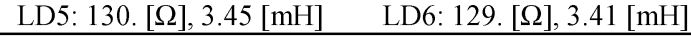

SVC: the switched inductance $L$, the fixed capacitance $C$, and the firing delay angle $\alpha$ of the SVC.

$L=995[\mathrm{mH}], C=7.07[\mu \mathrm{F}], \alpha=29.5^{\circ}$

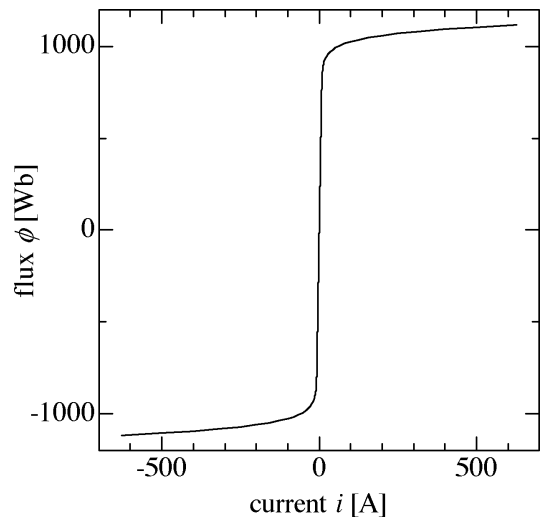

Fig. 5. Current-flux $(i-\phi)$ curve of the transformer magnetizing branches.

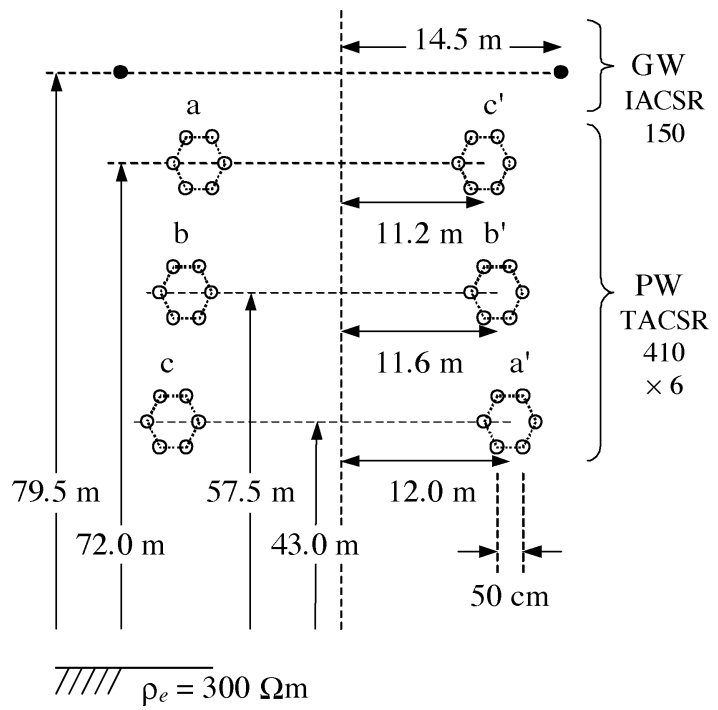

Fig. 6. Conductor configuration of the $500 \mathrm{kV}$ double-circuit transmission lines.

though Fig. 4(a) is shown on a one-line diagram, all components are represented by three-phase models.

In this test case, the generators are represented by balanced three-phase sinusoidal voltage sources. The transformers are represented by the simplified equivalent shown in Fig. 4(b) with 


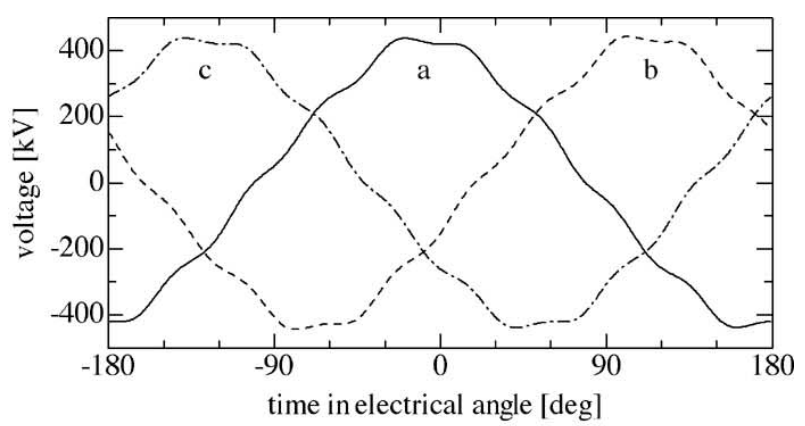

(a)
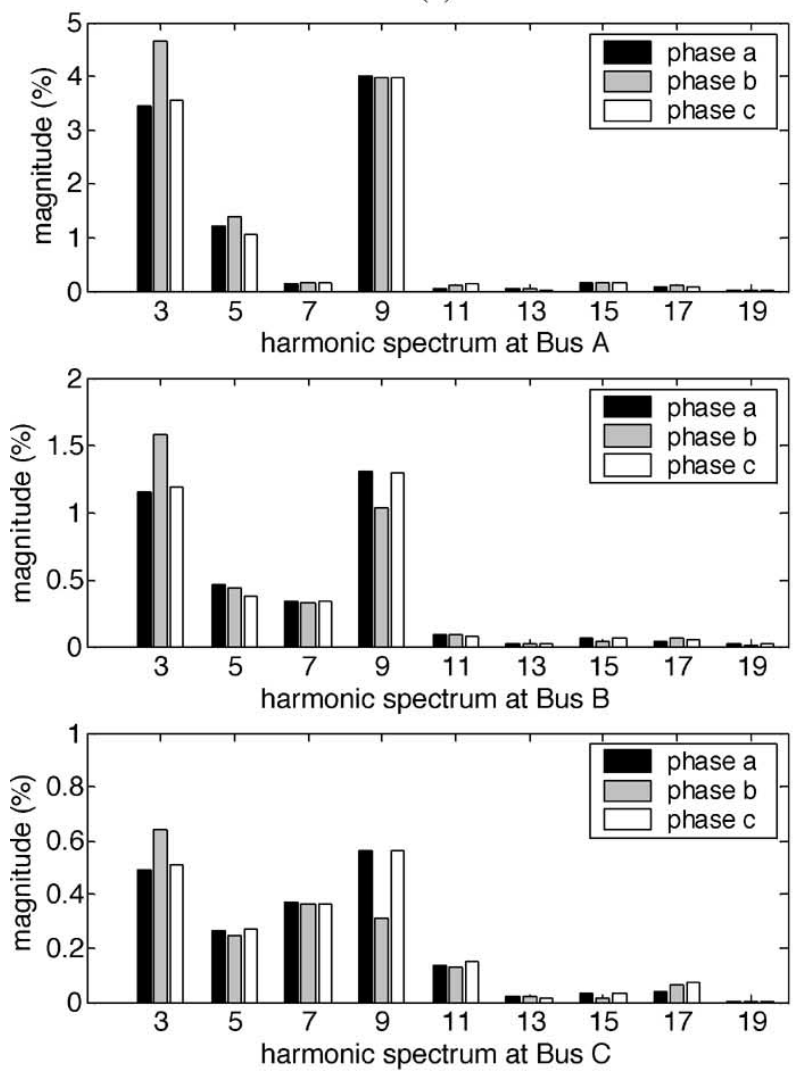

(b)

Fig. 7. The calculated voltage waveforms at Bus A are shown in (a). The harmonic spectra of the voltages at Buses A-C are shown in (b) (the magnitudes are normalized by the corresponding fundamental components). (a) Voltage waveforms at Bus A; (b) harmonic spectra of voltages at Buses A-C.

the leakage inductances $L_{0}$ and the magnetizing branches $L_{N}$. All magnetizing branches have the same saturation characteristic defined by

$$
i=0.01 \phi+5 \times 10^{-68} \phi^{23}
$$

where $i$ is the current and $\phi$ the flux, and the current-flux $(i-\phi)$ curve is shown in Fig. 5. The transmission lines are all untransposed and have the same conductor configuration shown in Fig. 6. At both ends of the transmission lines phases a and $\mathrm{a}^{\prime}$, $\mathrm{b}$ and $\mathrm{b}^{\prime}$, and $\mathrm{c}$ and $\mathrm{c}^{\prime}$ are connected, and thus the admittance is given by the $6 \times 6$ matrix

$$
\left[\begin{array}{cc}
Y_{0}(\omega) \operatorname{coth}(\Gamma(\omega) l) & -Y_{0}(\omega) \operatorname{cosech}(\Gamma(\omega) l) \\
-Y_{0}(\omega) \operatorname{cosech}(\Gamma(\omega) l) & Y_{0}(\omega) \operatorname{coth}(\Gamma(\omega) l)
\end{array}\right]
$$

where $Y_{0}(\omega)$ is the $3 \times 3$ characteristic admittance matrix, $\Gamma(\omega)$ the $3 \times 3$ propagation constant matrix, and $l$ the length. $Y_{0}(\omega)$

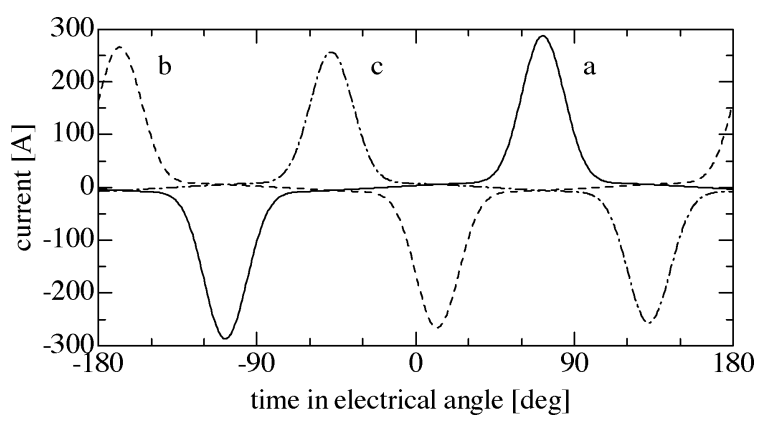

Fig. 8. Calculated current waveforms through the nonlinear inductors of TR1.

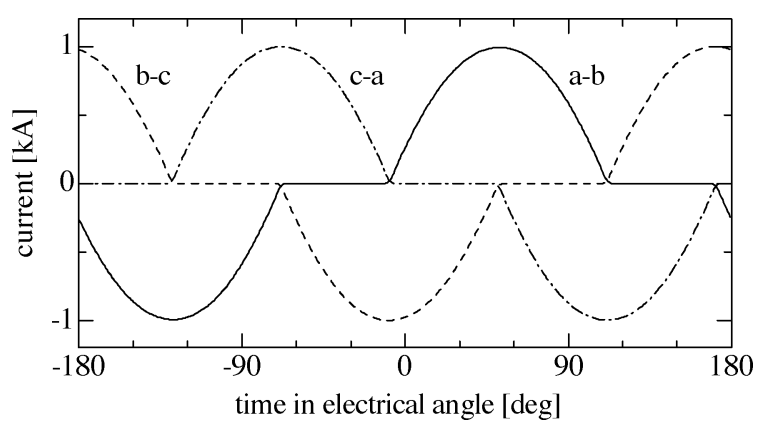

Fig. 9. Calculated current waveforms through the inductors of the SVC.

and $\Gamma(\omega)$ are calculated considering full frequency dependence [18], [19]. Substituting (55) into (25), the HDAMs of the transmission lines are obtained. The loads are represented by the $R-L$ series circuit shown in Fig. 4(c). The SVC shown in Fig. 4(d) is an FC-TCR type and the HDAM of each thyristor-inductor branch is given by (32).

With up to the \pm 49 th harmonic component the solution is calculated considering half-wave symmetry. The initial solution is obtained by solving $J(0) z^{(0)}=-u$. Fig. 7(a) shows the voltage waveforms at Bus A which is distorted by the SVC, and Fig. 7(b) the harmonic spectra of the voltages at Buses A-C. It is observed that the harmonics generated by the SVC propagate along the transmission lines. Fig. 8 shows the magnetizing currents of TR1. The magnetizing currents of TR2-4 are similar to those of TR1. The imbalanced impedances of the untransposed transmission lines create the different peak values of the magnetizing currents phase by phase, and this imbalance can be observed also in Fig. 7(b). Fig. 9 shows the currents through the inductors of the SVC.

The Newton-Raphson iteration is terminated when the relative norm of the correction term $\Delta z^{(m)}$ becomes less than 10 times of machine epsilon $\varepsilon$ :

$$
\frac{\left\|\Delta z^{(m)}\right\|_{2}}{\left\|z^{(m)}\right\|_{2}}<10 \varepsilon
$$

The variation of the relative norm of the correction term during the iteration is shown in Fig. 10. $\varepsilon$ of the computer used is $2.22 \times$ $10^{-16}$, and (56) is satisfied at the fifth step. The convergence rate is observed to be quadratic. Reference [20] compares the convergence rates of various iteration schemes and shows that a quadratic convergence is the fastest.

The size of the Jacobian matrix $J$ is $2400 \times 2400$, and its sparsity pattern is shown in Fig. 11. Sub-blocks corresponding 


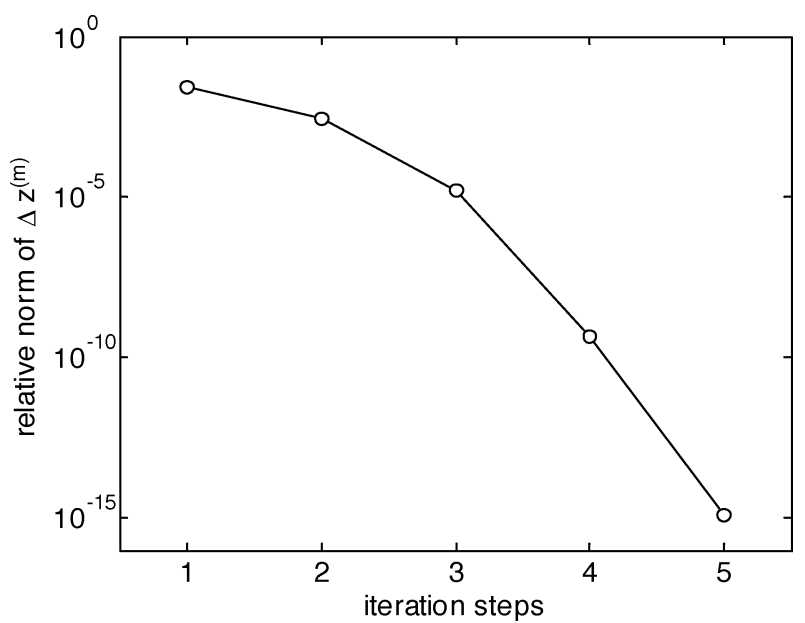

Fig. 10. Relative norm of the correction term $\Delta z^{(m)}$ with respect to iteration steps $m$.

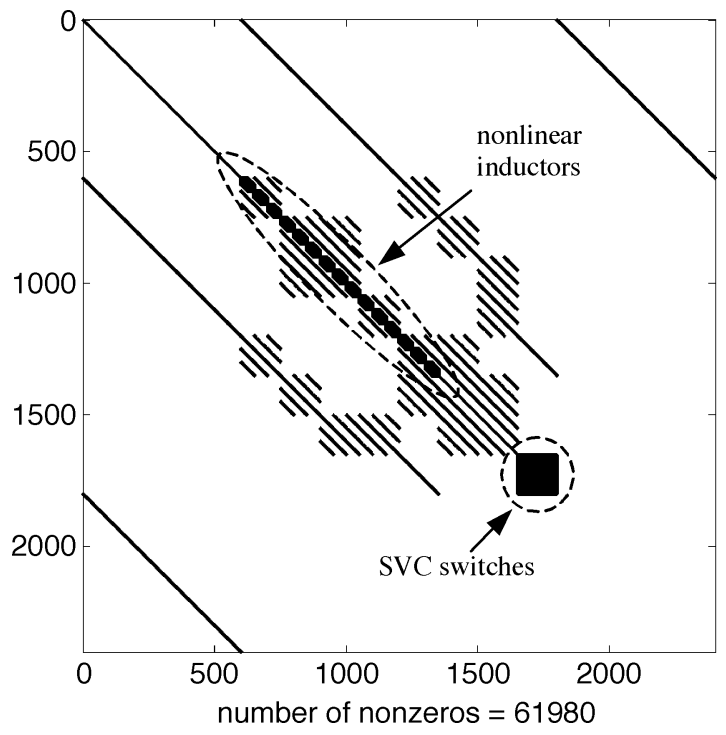

Fig. 11. Sparsity pattern of Jacobian matrix.

to the HDAMs of linear elements have a diagonal pattern, because there are no couplings among harmonics of different orders. On the other hand, sub-blocks corresponding to nonlinear and switching elements show patterns of couplings among different orders of harmonics. To store $J$ in memory and to solve (52), the sparsity of $J$ was taken into account [21]. With the sparse matrix technique, the storage required was $1.2 \mathrm{MB}$ and the computation time for this simulation was 19.5 seconds using a personal computer with Pentium $4 \mathrm{CPU}(2.4 \mathrm{GHz})$. These requirements are acceptable for practical usage. The program is coded using MATLAB ver. 6.5.

\section{CONCLUSIONS}

In this paper an entirely harmonic domain algorithm for obtaining the periodic steady-state solution of a multiphase network with nonlinear, switching, and frequency dependent elements is presented. The proposed Newton-Raphson iteration process takes rigorously into account the couplings among different harmonics and a quadratic convergence rate is achieved.

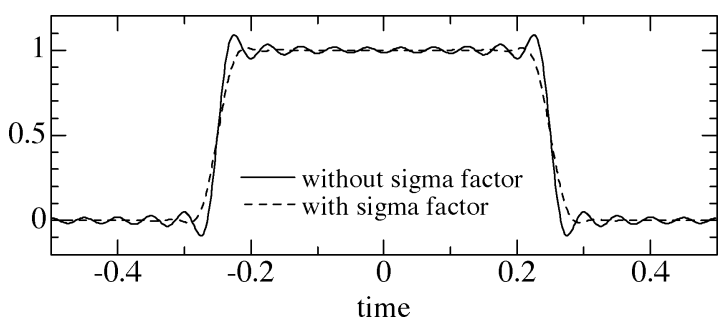

Fig. 12. Calculated results with and without the sigma factor window.

By extending the modified nodal equations approach to the harmonic domain case, the network equations can always be derived regardless of network topology, and the network elements are modeled in a modular approach. The algorithm was applied to obtain the periodic steady-state solution of a $500 \mathrm{kV}$ double-circuit three-phase network with nonlinear inductors, an SVC, and frequency dependent transmission lines.

\section{APPENDIX}

\section{A. Effectiveness of Sigma Factor Window}

To show the effectiveness of the Sigma Factor window [15], the function

$$
p(t)= \begin{cases}1, & (-0.5<t<0.5) \\ 0, & \text { otherwise }\end{cases}
$$

is reproduced with its Fourier coefficients

$$
c_{k}=\frac{\sin \left(k \frac{\pi}{2}\right)}{k \pi} \quad(k=0, \pm 1, \pm 2, \cdots)
$$

only up to the \pm 19 th with and without the Sigma Factor window. As shown in Fig. 12, the Sigma Factor eliminates the oscillation called the Gibbs phenomenon and gives a better approximation to the original function.

\section{ACKNOWLEDGMENT}

The authors would like to thank Dr. L. T. G. Lima of Power Technologies, Inc. (formerly Visiting Scientist at the University of Toronto) and Dr. M. Takasaki of CRIEPI for useful discussions.

\section{REFERENCES}

[1] D. Xia and G. T. Heydt, "Harmonic power flow studies Part I-Formulation and solution," IEEE Trans. Power App. Syst., vol. PAS-101, pp. 1257-1265, June 1982.

[2] — , "Harmonic power flow studies Part II-Implementation and practical application," IEEE Trans. Power App. Syst., vol. PAS-101, pp. 1266-1270, June 1982.

[3] T. J. Densem, P. S. Bodger, and J. Arrillaga, "Three phase transmission system modeling for harmonic penetration studies," IEEE Trans. Power App. Syst., vol. PAS-103, pp. 310-317, Feb. 1984.

[4] A. Semlyen, E. Acha, and J. Arrillaga, "Newton-type algorithms for the harmonic phasor analysis of nonlinear power circuits in periodical steady state with special reference to magnetic nonlinearities," IEEE Trans. Power Delivery, vol. 3, pp. 1090-1098, July 1988.

[5] W. Xu, J. R. Martí, and H. W. Dommel, "A multiphase harmonic load flow solution technique," IEEE Trans. Power Syst., vol. 6, pp. 174-182, Feb. 1991.

[6] X. Lombard, J. Mahseredjian, S. Lefebvre, and C. Kieny, "Implementation of a new harmonic initialization method in the EMTP," IEEE Trans. Power Delivery, vol. 10, pp. 1343-1352, July 1995.

[7] G. Murere, S. Lefebvre, and X. Dai Do, "A generalized harmonic balance method for EMTP initialization," IEEE Trans. Power Delivery, vol. 10, pp. 1353-1359, July 1995. 
[8] G. N. Bathurst, B. C. Smith, N. R. Watson, and J. Arrillaga, "A modular approach to the solution of the three-phase harmonic power-flow," IEEE Trans. Power Delivery, vol. 15, pp. 984-989, July 2000.

[9] B. K. Perkins, J. R. Martí, and H. W. Dommel, "Nonlinear elements in the EMTP: steady-state initialization," IEEE Trans. Power Syst., vol. 10, pp. 593-601, May 1995.

[10] Q. Wang and J. R. Martí, "A waveform relaxation technique for steady state initialization of circuits with nonlinear elements and ideal diodes," IEEE Trans. Power Delivery, vol. 11, pp. 1437-1443, July 1996.

[11] A. Semlyen and A. Medina, "Computation of the periodic steady-state in systems with nonlinear components using a hybrid time and frequency domain methodology," IEEE Trans. Power Syst., vol. 10, pp. 1498-1504, Aug. 1995.

[12] A. Semlyen and M. Shlash, "Principles of modular harmonic power flow methodology," Proc. Inst. Elect. Eng., Gen. Transm. Dist., vol. 147, no. 1, pp. 1-6, Jan. 2000.

[13] C.-W. Ho, A. E. Ruehli, and P. A. Brennan, "The modified nodal approach to network analysis," IEEE Trans. Circuit Syst., vol. CAS-22, pp. 504-509, June 1975.

[14] E. Acha and M. Madrigal, Power System Harmonics-Computer Modeling and Analysis. New York: Wiley, 2001.

[15] S. J. Day, N. Mullineux, and J. R. Reed, "Developments in obtaining transient response using Fourier Transform," Int. J. Elect. Eng. Educ., vol. 3, pp. 501-506, 1965.

[16] L. T. G. Lima, A. Semlyen, and R. Iravani, "Harmonic domain periodic steady state modeling of power electronics apparatus: SVC and TCSC," IEEE Trans. Power Delivery, vol. 18, pp. 960-967, July 2003.

[17] W. J. McCalla, Fundamentals of Computer-Aided Circuit Simulation. Norwell, MA: Kluwer, 1988

[18] J. R. Carson, "Wave propagation in overhead wires with ground return," Bell Syst. Tech. J., vol. 5, pp. 539-554, 1926.

[19] S. A. Schelkunoff, "The electromagnetic theory of coaxial transmission line and cylindrical shields," Bell Syst. Tech. J., vol. 13, pp. 532-579, 1934.

[20] L. T. G. Lima, A. Semlyen, and R. Iravani, "Fast initialization for transient calculations with nonsinusoidal steady state," in Proc. Int. Conf. Power Syst. Transients, Rio de Janeiro, Brazil, June 2001, pp. 369-374.

[21] Using MATLAB (Version 6). Natick, MA: The MathWorks, Inc., 2000 sec. 16.
Taku Noda (M'97) was born in Osaka, Japan, on July 4, 1969. He received the B.S., M.S., and Ph.D. degrees in engineering from Doshisha University, Kyoto, Japan, in 1992, 1994, and 1997, respectively.

He was with DEI Simulation Software, Neskowin, OR, in 1994, and a Consultant with Bonneville Power Administration (BPA), Portland, OR, in 1995. In 1997, he joined Central Research Institute of Electric Power Industry (CRIEPI), where he is currently a Research Scientist. From 2001 to 2002, he was a Visiting Scientist at the University of Toronto, Toronto, ON, Canada. His main research interest is the transient analysis of power systems.

Dr. Noda is a member of IEE and IEE of Japan.

Adam Semlyen (LF'97) was born in 1923 in Rumania. He received the Dipl.Ing. degree from the Polytechnic Institute of Timisoara, Romania, in 1950, and the Ph.D. degree from the Polytechnic Institute of Iasi, Romania, in 1965.

He started his career in Timisoara with an electric power utility and held academic positions at the Polytechnic Institute. In 1969, he joined the University of Toronto, Toronto, ON, Canada, where he is a Professor in the Department of Electrical and Computer Engineering, emeritus since 1988. His research interests include steady state and dynamic analysis as well as computation of electromagnetic transients in power systems.

Reza Iravani (F'03) received the B.Sc. degree in electrical engineering from Tehran Polytechnique University, Tehran, Iran, in 1976, and the M.Sc. and Ph.D. degrees in electrical engineering from the University of Manitoba, Winnipeg, MB, Canada, in 1981 and 1985, respectively.

Currently, he is a Professor at the University of Toronto, Toronto, ON, Canada. His research interests include power electronics and power system dynamics and control. 